\title{
Comparison of Kernel Wax from Corn Genotypes Resistant or Susceptible to Aspergillus flavus
}

\author{
J. S. Russin, B. Z. Guo, K. M. Tubajika, R. L. Brown, T. E. Cleveland, and N. W. Widstrom
}

First, second, and third authors: Department of Plant Pathology and Crop Physiology, Louisiana State University Agricultural Center, Baton Rouge 70803; fourth and fifth authors: U.S. Department of Agriculture, Agricultural Research Service, Southern Regional Research Center, New Orleans, LA 70179; and sixth author: U.S. Department of Agriculture, Agricultural Research Service, Insect Biology and Population Management Research Laboratory, Tifton, GA 31793. Accepted for publication 31 January 1997.

\begin{abstract}
Russin, J. S., Guo, B. Z., Tubajika, K. M., Brown, R. L., Cleveland, T. E., and Widstrom, N. W. 1997. Comparison of kernel wax from corn genotypes resistant or susceptible to Aspergillus flavus. Phytopathology 87: 529-533.

Kernels of corn genotype GT-MAS : gk are resistant to Aspergillus flavus. Earlier studies showed that this resistance is due in part to kernel pericarp wax. Experiments were conducted to compare wax from GTMAS : gk kernels with that from kernels of several susceptible commercial hybrids. GT-MAS : gk had more pericarp wax than did the susceptible hybrids. Scanning electron microscopy revealed that GT-MAS : gk kernels

Susceptible kernels appeared much more smooth and lacked the abundant surface deposits observed in GT-MAS : gk. In vitro bioassays showed that kernel wax from GT-MAS : gk reduced A. flavus colony diameter by $35 \%$. Colony diameters on a medium amended with wax from susceptible kernels did not differ from those of controls. Thin-layer chromatography and analyses of chromatograms using NIH Image software showed a distinctive composition for GT-MAS:gk kernel wax. Chromatograms of wax from GT-MAS:gk contained a peak unique to this genotype, but also lacked a peak common to all susceptible hybrids. This is the first report of specific kernel factors involved in resistance to A. flavus in corn.
\end{abstract} appeared rough and showed abundant wax deposits on kernel surfaces.
Additional keywords: food safety, mycotoxin, pericarp, Zea mays.
Contamination of food and feed grains by aflatoxins is a problem both in the United States and throughout the world. Aflatoxins, which are secondary metabolites produced by the fungi Aspergillus flavus Link:Fr. and A. parasiticus Speare, are potent carcinogens and can pose a serious health hazard when they contaminate food for poultry, livestock, or humans $(7,11,16)$. Aflatoxin B1 is the most potent naturally occurring carcinogenic substance known (24). Aflatoxin contamination of corn (Zea mays L.), caused by A. flavus, occurs occasionally in the midwestern United States, but is a chronic problem in the southeastern states (9). Yield losses are minimal, but value losses can be severe, because high levels of aflatoxin can render grain unmerchantable (22).

Since the first report of aflatoxin contamination in preharvest corn fields (2), substantial effort has been made to identify corn genotypes that resist infection by A. flavus. Although differences between corn genotypes have been demonstrated $(10,17,28)$, relatively few show significant levels of resistance. Widstrom et al. (27) reported that the corn population GT-MAS:gk consistently showed resistance when compared with susceptible hybrids. In repeated tests, fungus colonization in individual inoculated kernels of GT-MAS:gk was reduced, and aflatoxin levels in this genotype were several orders of magnitude lower than those in susceptible commercial hybrids $(12,14)$. Although mechanisms of resistance in this genotype are not completely understood, Guo et al. (14) showed that resistance is associated with a preformed compound or compounds within the kernel, as well as wax

Corresponding author: J. S. Russin; E-mail address: jrussin@1suvm.sncc.lsu.edu

Publication no. P-1997-0303-01R

(c) 1997 The American Phytopathological Society and cutin in the intact kernel pericarp. The objective of this research was to compare kernel wax from resistant and susceptible corn genotypes with regard to amount, appearance, antifungal activity, and composition. Preliminary reports have been published $(12,26)$.

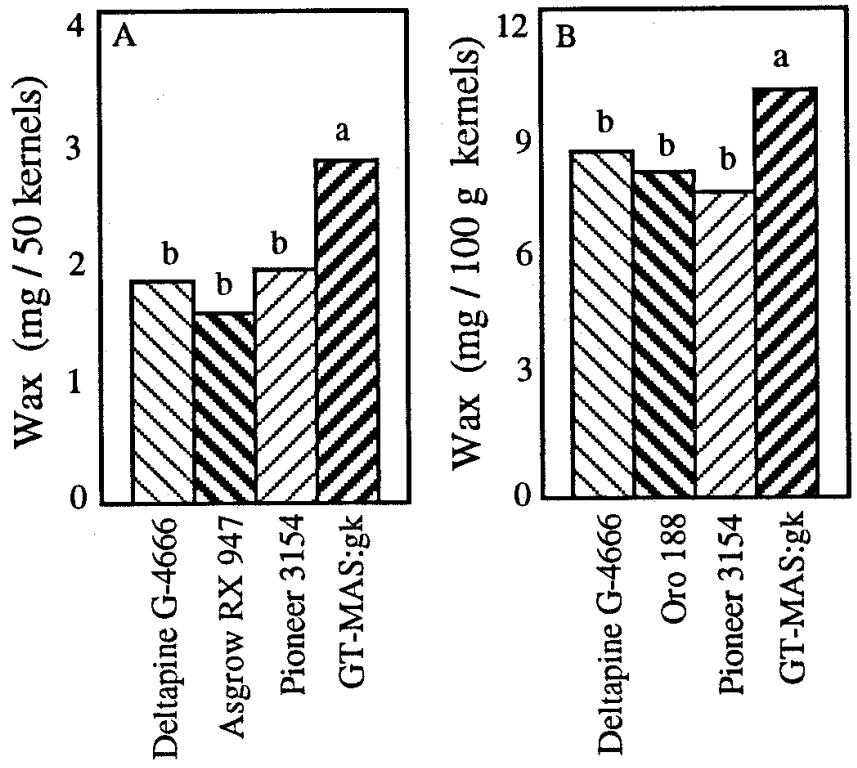

Fig. 1. Pericarp wax from kernels of corn genotype GT-MAS:gk and commercial hybrids Deltapine G-4666, Asgrow RX 947 or Oro 188, and Pioneer 3154. Wax was removed from $\mathbf{A}$, kernels produced as grain following open pollination and $\mathbf{B}$, kernels provided as seed. In each graph, treatment means labeled with the same letter did not differ significantly $(P>0.05)$ according to least significant difference. 


\section{MATERIALS AND METHODS}

General procedures. Commercial corn hybrids Asgrow RX 947, Deltapine G-4666, Oro 188, and Pioneer 3154 were provided by their respective companies. Controlled laboratory inoculations, as well as field studies, showed that these hybrids are susceptible to colonization by A. flavus and subsequent accumulation of aflatoxin B1 (14; B. Z. Guo, K. M. Tubajika, and J. S. Russin, unpublished data). In some experiments, Oro 188 replaced Asgrow RX 947, because the latter was not available. Corn population GT-MAS:gk is resistant to A. flavus colonization and aflatoxin accumulation (6, 14). Kernels of all genotypes were maintained at $4^{\circ} \mathrm{C}$ and were free of fungicides, insecticides, and dyes normally applied prior to sale. Intact kernels of uniform size and shape were used in all studies.

Strain AF13 of A. flavus, which produces abundant aflatoxin B1 $(5,8)$, was provided by P. J. Cotty (USDA/ARS/SRRC, New Orleans). The fungus was grown on V8 juice agar plates (5\% V8 juice and $2 \%$ agar) at $28^{\circ} \mathrm{C}$ in darkness. Inoculum was conidia obtained from 7-day-old cultures and suspended in water.

Amount of kernel wax. Kernels from two sources were used in these studies. Experiment 1 examined amounts of wax on kernels produced in the field as grain. Plots of Asgrow RX 947, Deltapine G-4666, Pioneer 3154, and GT-MAS:gk were established in Winnsboro, LA, in 1993. The plots were furrow-irrigated as needed to minimize moisture stress. At maturity, ears from these plots were harvested and shelled by hand to eliminate the physical da- mage to kernel pericarps that can occur during mechanical harvest. In tared flasks, 50 kernels of each genotype were immersed in 100 $\mathrm{ml}$ of chloroform for $60 \mathrm{~s}$. Care was taken to clean the kernels of all debris prior to immersion. The kernels were removed, the chloroform was evaporated to dryness, and the weight of the wax from each genotype was determined. The genotypes were replicated four times, and the experiment was conducted twice. Experiment 2 examined amounts of wax on kernels supplied as seed. In tared flasks, $100 \mathrm{~g}$ of kernels of corn genotypes Deltapine G-4666, Oro 188, Pioneer 3154, and GT-MAS : gk were immersed in $200 \mathrm{ml}$ of chloroform for $60 \mathrm{~s}$. The kernels were removed, the chloroform was evaporated to dryness, and the weight of the wax from each genotype was determined. The genotypes were replicated five times, and the experiment was conducted three times.

Scanning electron microscopy of kernel wax. Corn genotypes Asgrow RX 947, Deltapine G-4666, Pioneer 3154, and GTMAS : gk supplied as seed were used in this study. Ten kernels of each genotype were prepared for scanning electron microscopy by desiccation for $72 \mathrm{~h}$. Intact kernels of uniform size and shape were mounted (endosperm side up) on aluminum stubs, coated with gold-palladium (20 nm thick) applied with an Edwards S-150 sputter coater, and examined with a Leica Cambridge S 260 scanning electron microscope.

Bioassay of kernel wax. Kernels (100 g) of corn genotypes Deltapine G-4666, Oro 188, Pioneer 3154, and GT-MAS : gk supplied as seed were immersed in $200 \mathrm{ml}$ of chloroform for $60 \mathrm{~s}$.
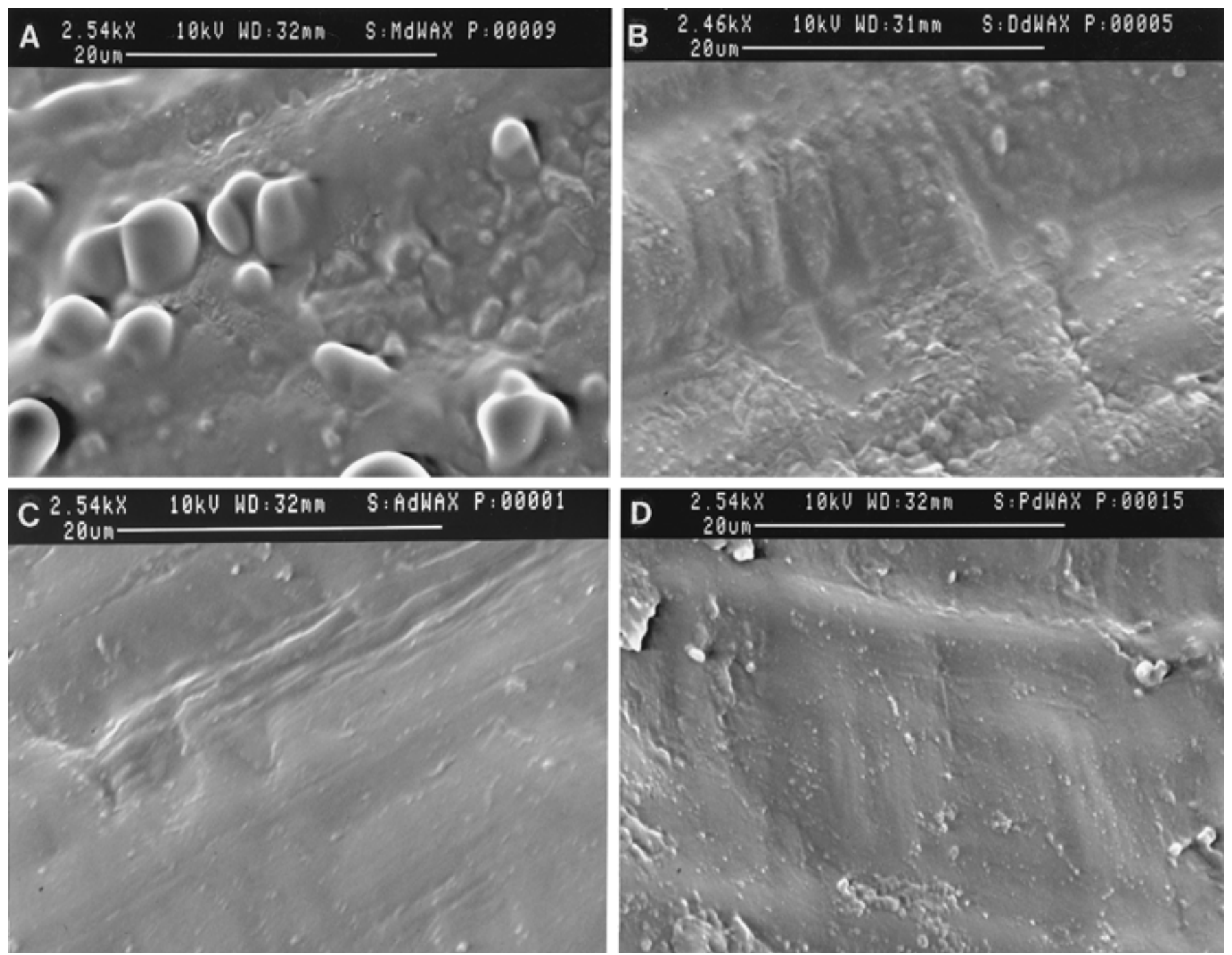

Fig. 2. Scanning electron micrographs of kernel surfaces of A, corn genotype GT-MAS:gk; and commercial hybrids B, Deltapine G-4666; C, Asgrow RX 947; and $\mathbf{D}$, Pioneer 3154 
The kernels were removed, the chloroform was evaporated to dryness, and the residual wax from each genotype was redissolved in $3 \mathrm{ml}$ of chloroform. This was combined with autoclaved A\&M medium (1) to make a final volume of $100 \mathrm{ml}$. The mixture was poured into petri dishes, which were covered loosely for $2 \mathrm{~h}$ before inoculation to allow the chloroform to evaporate. Wax concentrations in Deltapine G-4666, Oro 188, Pioneer 3154, and GTMAS:gk were approximately $0.085,0.08,0.075$, and $0.1 \mathrm{mg} / \mathrm{ml}$, respectively. The plates were then inoculated with $A$. flavus; $10 \mu \mathrm{l}$ of a conidial suspension $\left(10^{6}\right.$ conidia per $\left.\mathrm{ml}\right)$ was placed in the center of each plate. Controls were A\&M medium alone and A\&M medium amended with chloroform at the same concentration. The plates were incubated at $28^{\circ} \mathrm{C}$ in darkness, and colony diameter was measured after $24 \mathrm{~h}$. The treatments were replicated five times, and the experiment was conducted twice.

Thin-layer chromatography of kernel wax. Kernels (150 g) of corn genotypes Deltapine G-4666, Oro 188, Pioneer 3154, and GT-MAS:gk were immersed in $200 \mathrm{ml}$ of chloroform for $60 \mathrm{~s}$ to remove wax. The chloroform was evaporated to dryness under a stream of nitrogen. The residual wax was dissolved in $1 \mathrm{ml}$ of chloroform, evaporated again under nitrogen, and redissolved in 1 $\mathrm{ml}$ of hexane. Samples (10 and $20 \mu \mathrm{l})$ from each corn genotype were spotted on silica gel thin-layer chromatography plates ( 20 by $20 \mathrm{~cm}$ ). The plates were developed at ambient temperature (20 to $25^{\circ} \mathrm{C}$ ) in glass tanks by the ascending solvent technique. Solvent systems were methylene chloride, benzene/chloroform (7:3 vol/ vol), and chloroform/ethyl acetate (1:1 vol/vol) (20). To make wax components visible, the chromatograms were sprayed with concentrated sulfuric acid (15). Before the plates were completely charred by acid, those developed in benzene/chloroform were photocopied, and the copies were scanned into a computer. The images were then analyzed with NIH Image software (version 1.54) to determine the relative density and location of spots on the chromatograms. This software is in the public domain.

Statistical analysis. Data from wax weight determinations and wax bioassays were analyzed with the analysis of variance proce-

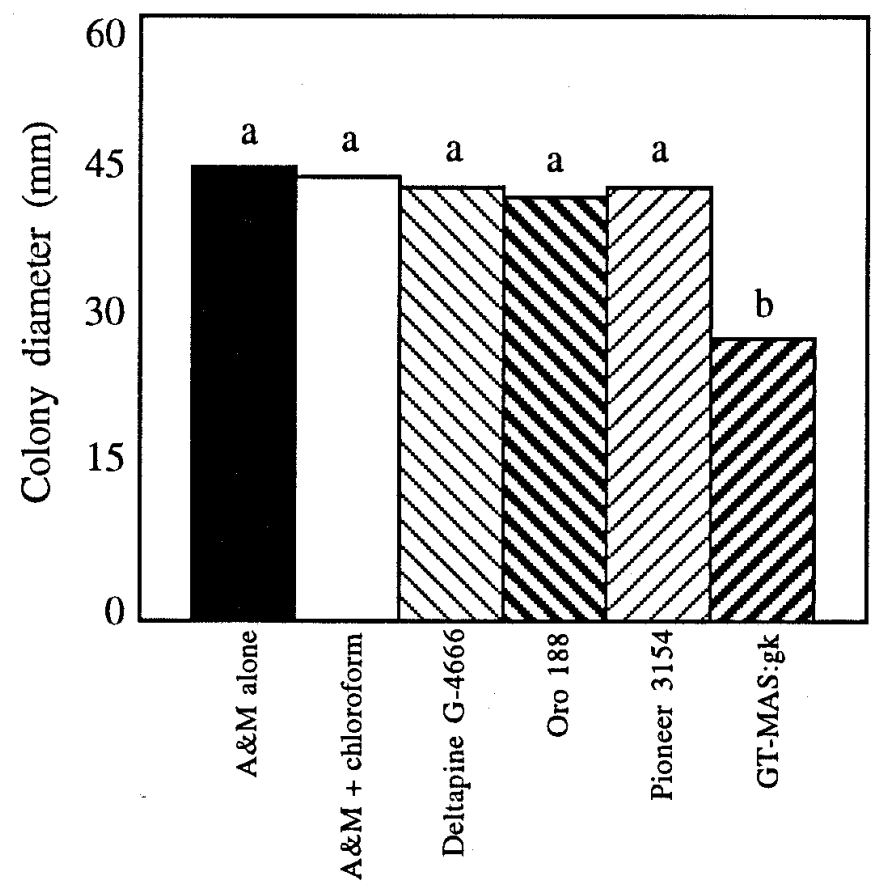

Fig. 3. Diameters of Aspergillus flavus colonies on A\&M medium alone, amended with chloroform, or amended with chloroform containing kernel pericarp wax from corn genotype GT-MAS:gk or commercial hybrids Deltapine G-4666, Oro 188, or Pioneer 3154. Treatment means labeled with the same letter did not differ significantly $(P>0.05)$ according to least significant difference. dure of Statistical Analysis System (SAS Institute, Cary, NC). Means were separated by least significant difference $(P \leq 0.05)$.

\section{RESULTS}

Analyses showed no significant test-by-treatment interactions for kernel wax weight. Therefore data from duplicate tests were combined for final analysis. Kernels of resistant GT-MAS : gk had significantly more pericarp wax than did the susceptible hybrids tested (Fig. 1).

Scanning electron microscopy revealed distinct differences between kernels of resistant and susceptible corn genotypes (Fig. 2). Resistant GT-MAS:gk kernels were rough in appearance and had abundant wax deposits on kernel surfaces. Asgrow RX 947, Deltapine G-4666, and Pioneer 3154 kernels appeared much more smooth and lacked the abundant surface deposits observed on GTMAS : gk.

No significant test-by-treatment interactions were detected for wax bioassay data. Therefore, data from duplicate tests were combined for final analysis. Bioassays showed that only kernel wax from the resistant GT-MAS: gk reduced A. flavus colony diameter (Fig. 3). This reduction averaged about 35\% across tests. Colony diameters of $A$. flavus on media amended with kernel wax from susceptible hybrids did not differ from those of the controls (Fig. 3).

Thin-layer chromatography showed differences in the kernel wax composition of the corn genotypes tested in all three solvent systems. A band unique to GT-MAS:gk had $R_{f}$ values of 0.30 , 0.81 , and 0.40 in benzene/chloroform ( $7: 3 \mathrm{vol} / \mathrm{vol})$, chloroform/ ethyl acetate $(1: 1 \mathrm{vol} / \mathrm{vol})$, and methylene chloride, respectively. Because benzene/chloroform provided the best separation of kernel wax components (Fig. 4), this chromatogram was analyzed with NIH Image software. Seven peaks were identified. On the basis of $R_{f}$ values, these peaks were designated P1 $\left(R_{f}=0.07\right), \mathrm{P} 2$ $\left(R_{f}=0.14\right), \mathrm{P} 3\left(R_{f}=0.21\right), \mathrm{P} 4\left(R_{f}=0.25\right), \mathrm{P} 5\left(R_{f}=0.30\right), \mathrm{P} 6\left(R_{f}=\right.$ $0.48)$, and P7 $\left(R_{f}=0.91\right)$ (Fig. 5). P1 and P2 were present in the chromatograms of all genotypes, but at lower density in that of the resistant GT-MAS : gk. P7 was present in the chromatograms of all genotypes at approximately equal densities. P3 and P4 were present only in the chromatograms of Deltapine G-4666 and GT-MAS:

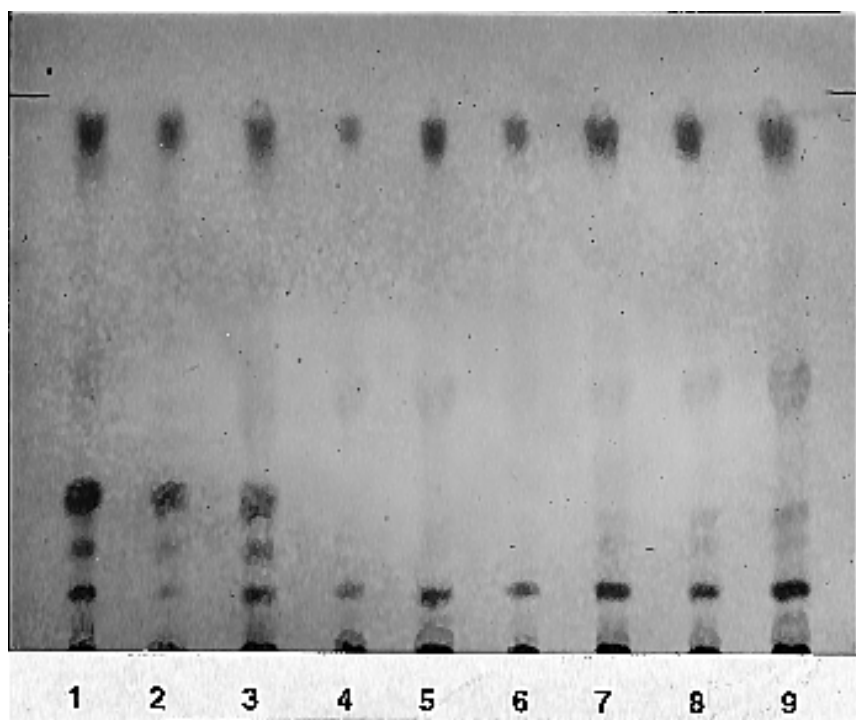

Fig. 4. Thin-layer chromatograms of corn kernel pericarp wax developed in benzene/chloroform (7:3 vol/vol): lanes $1(30 \mu \mathrm{l}), 2(15 \mu \mathrm{l})$, and $3(30 \mu \mathrm{l})$ from GT-MAS:gk; lanes $4(15 \mu \mathrm{l})$ and $5(30 \mu \mathrm{l})$ from Pioneer 3154; lanes 6 $(15 \mu \mathrm{l})$ and $7(30 \mu \mathrm{l})$ from Oro 188 ; and lanes $8(15 \mu \mathrm{l})$ and $9(30 \mu \mathrm{l})$ from Deltapine G-4666. The chromatograms were sprayed with concentrated sulfuric acid to make the spots visible. 
gk. Of particular interest were P5, present only in the chromatogram of GT-MAS : gk, and P6, present in the chromatograms of all genotypes except GT-MAS :gk (Fig. 5).

\section{DISCUSSION}

The pericarp is the outermost portion of corn kernels and is composed of several layers of cells differing in chemical components and cell wall thickness (29). The pericarp contains layers of cutin and wax, which afford considerable protection against infection by fungal pathogens $(19,21)$. Several researchers have shown that the pericarp is important for protecting seeds of certain

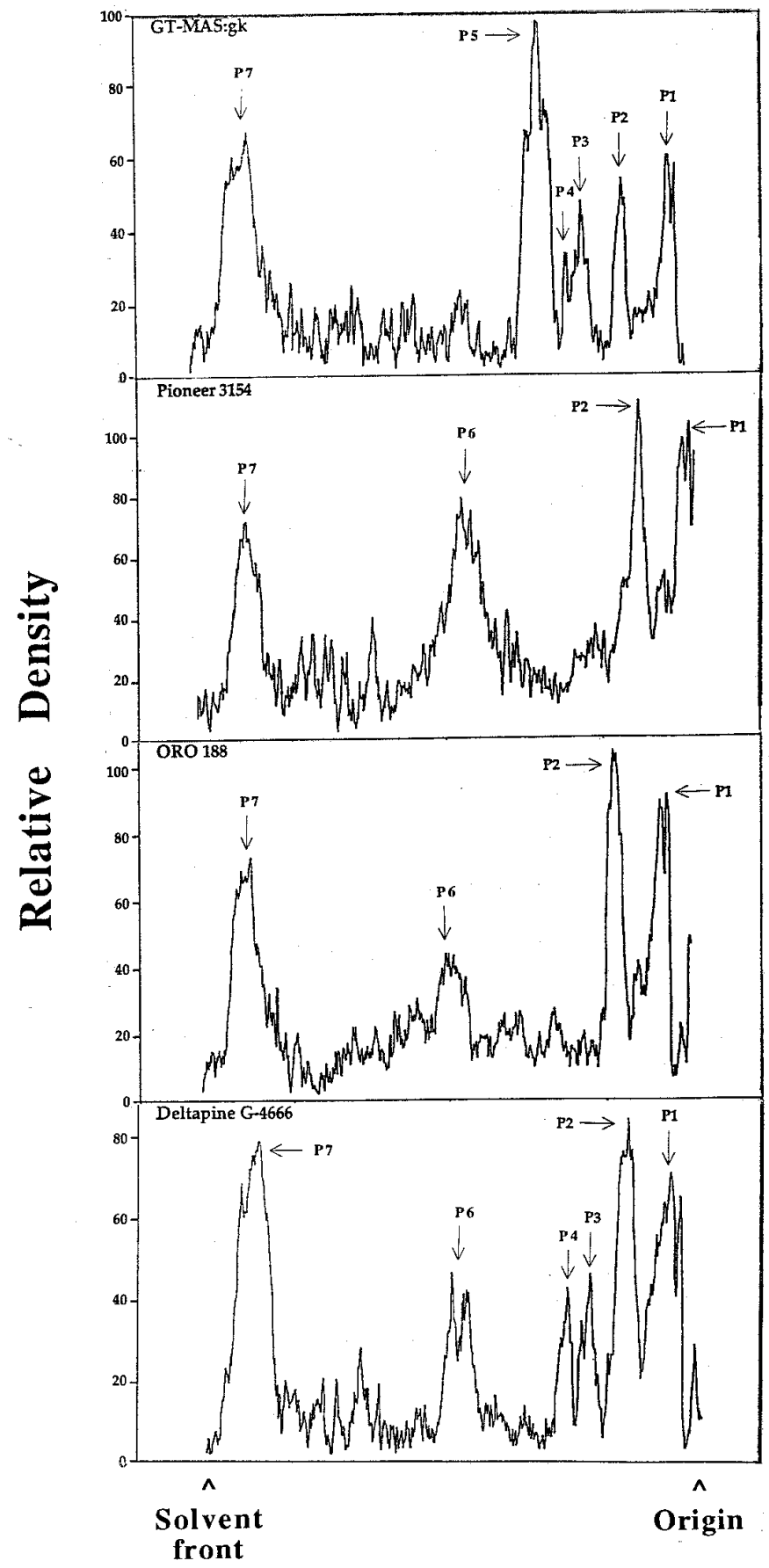

Fig. 5. Relative densities of separated components from thin-layer chromatograms of kernel pericarp wax from corn genotype GT-MAS:gk and commercial hybrids Pioneer 3154, Oro 188, and Deltapine G-4666. Seven peaks (P1P7) were identified among the four genotypes. crop species from A. flavus infection and subsequent aflatoxin accumulation $(23,25)$. Although no specific mechanisms were mentioned, Reddy et al. (23) suggested a role for seed surface wax in this resistance. We showed previously (14) that removing kernel wax prior to inoculation with $A$. flavus raised the aflatoxin level significantly in the resistant corn genotype GT-MAS:gk, but not in a susceptible commercial hybrid. This suggested a direct role for kernel wax in the resistance of GT-MAS:gk to A. flavus. Results from our present study support this previous work and provide further evidence for kernel wax as an important factor in corn kernel resistance to A. flavus.

Direct comparisons of wax weight data, as well as scanning electron micrographs, indicate that kernels of GT-MAS:gk had consistently more surface wax than did susceptible commercial hybrids tested. This indicates that resistance to A. flavus in GTMAS : gk kernels may be due in part to a physical barrier provided by pericarp wax. Our results show further that kernel wax from this genotype was inhibitory to the growth of A. flavus in vitro, which suggests that resistance may also result from direct antifungal activity of the wax. We did not test for antitoxigenic activity in these studies. Little is known about antifungal effects of kernel wax. Koidsumi (18) reported antifungal activity against $A$. flavus in cuticular lipids from certain insects. The active constituents were free medium-chain-length saturated fatty acids, such as caprylic acid or capric acid.

Thin-layer chromatograms and analyses from NIH Image software showed a distinctive composition for GT-MAS:gk kernel wax. Chromatograms of this wax contained one peak (P5) unique to GT-MAS:gk, but also lacked a peak (P6) common to all susceptible hybrids. Research by Bianchi and co-workers on corn surface waxes shows that their chemical composition can vary greatly in different parts of the plant (3). These authors showed that kernel wax contains a high percentage of esters, but very small amounts of aldehydes and alcohols (3). The identities of compounds represented by P5 and P6 in the chromatograms were not determined in the present study, but work is in progress to characterize these compounds and test them for antifungal and antitoxigenic activity.

Contamination of food and feed grains by A. flavus and aflatoxin is a serious public health concern. There is great need to identify factors that confer resistance in corn and to incorporate that resistance into commercial hybrids. Results from the present study show that resistance in GT-MAS : gk is due in part to chemically unique kernel wax that provides physical and/or chemical barriers to A. flavus infection. This is the first report of specific kernel factors involved in resistance. Future efforts will focus on the role of kernel wax barriers in other resistant corn genotypes (4), as well as the potential for using kernel wax as a selectable marker for resistance in corn breeding.

\section{ACKNOWLEDGMENTS}

This project was supported by USDA Cooperative Agreement 586435-2-130 and by the Louisiana Soybean and Small Grain Research and Promotion Board. Approved for publication by the Director of the Louisiana Agricultural Experiment Station as manuscript 96-38-0278. We thank C. H. Carter and T. R. Gardner for technical assistance, P. J. Cotty for the A. flavus strain, R. A. Laine for introducing us to NIH Image, S. W. Matthews for scanning electron microscopy assistance, and Z. Y. Chen and S. R. Stetina for manuscript reviews.

\section{LITERATURE CITED}

1. Adye, J., and Mateles, R. I. 1964. Incorporation of labelled compounds into aflatoxin. Biochim. Biophys. Acta 86:418-420.

2. Anderson, H. W., Nehring, E. W., and Wichser, W. R. 1975. Aflatoxin contamination of corn in the field. J. Agric. Food Chem. 23:775-782.

3. Bianchi, G., Avato, P., and Salamini, F. 1984. Surface waxes from grain, leaves, and husks of maize (Zea mays L.). Cereal Chem. 61:45-47.

4. Brown, R. L., Cleveland, T. E., Payne, G. A., Woloshuk, C. P., Camp- 
bell, K. W., and White, D. G. 1995. Determination of resistance to aflatoxin production in maize kernels and detection of fungal colonization using an Aspergillus flavus transformant expressing Escherichia coli $\beta$ glucuronidase. Phytopathology 85:983-989.

5. Brown, R. L., Cotty, P. J., and Cleveland, T. E. 1991. Reduction in aflatoxin content of maize by atoxigenic strains of Aspergillus flavus. J. Food Prot. 54:623-626.

6. Brown, R. L., Cotty, P. J., Cleveland, T. E., and Widstrom, N. W. 1993. Living maize embryo influences aflatoxin production in maize. J. Food Prot. 56:967-971.

7. Cleveland, T. E., and Bhatnagar, D. 1992. Molecular strategies for reducing aflatoxin levels in crops before harvest. Pages 205-228 in: Molecular Approaches to Improving Food Quality and Safety. D. Bhatnagar and T. E. Cleveland, eds. Van Nostrand Reinhold, New York.

8. Cotty, P. J. 1989. Virulence and cultural characteristics of two Aspergillus flavus strains pathogenic on cotton. Phytopathology 79:808-814.

9. Diener, U. L., Cole, R. J., Sanders, T. H., Payne, G. A., Lee, L. S., and Klich, M. A. 1987. Epidemiology of aflatoxin formation by Aspergillus flavus. Annu. Rev. Phytopathol. 25:249-270

10. Gardner, C. A. C., Darrah, L. L., Zuber, M. S., and Wallin, J. R. 1987. Genetic control of aflatoxin production in maize. Plant Dis. 71:426-429.

11. Goldblatt, L. A. 1969. Aflatoxin: Scientific Background, Control, and Implications. Academic Press, New York.

12. Guo, B. Z., Brown, R. L., Cleveland, T. E., and Widstrom, N. W. 1996. Quantitative and qualitative differences in cuticle wax between kernels of maize genotypes resistant and susceptible to Aspergillus flavus. (Abstr.) Phytopathology 86:S71.

13. Guo, B. Z., Russin, J. S., Brown, R. L., Cleveland, T. E., and Widstrom, N. W. 1996. Resistance to aflatoxin contamination in corn as influenced by relative humidity and kernel germination. J. Food Prot. 59:276-281.

14. Guo, B. Z., Russin, J. S., Cleveland, T. E., Brown, R. L., and Widstrom, N. W. 1995. Wax and cutin layers in maize kernels associated with resistance to aflatoxin production by Aspergillus flavus. J. Food Prot. 58: 296-300.

15. Holloway, P. J., and Challen, S. B. 1966. Thin layer chromatography in the study of natural waxes and their constituents. J. Chromatogr. 25:336-346.

16. Hsieh, D. P. H. 1989. Potential human health hazards of mycotoxins.
Pages 69-80 in: Mycotoxins and Phycotoxins. S. Natori, K. Hashimoto, and Y. Ueno, eds. Elsevier, Amsterdam.

17. King, S. B., and Scott, G. E. 1982. Screening maize single crosses for resistance to preharvest infection of kernels by Aspergillus flavus. (Abstr.) Phytopathology 72:942.

18. Koidsumi, K. 1957. Antifungal action of cuticular lipids in insects. J. Insect Physiol. 1:40-51.

19. Kolattukudy, P. E. 1981. Structure, biosynthesis, and biodegradation of cutin and suberin. Annu. Rev. Plant Physiol. 32:539-567.

20. Maloney, P. J., Albert, P. J., and Tulloch, A. P. 1988. Influence of epicuticular waxes from white spruce and balsam fir on feeding behavior of the eastern spruce budworm. J. Insect Behav. 1:197-208.

21. Martin, J. T., and Juniper, B. E. 1970. The biosynthesis and development of cuticles. Pages 157-192 in: The Cuticles of Plants. J. T. Martin and B. E. Juniper, eds. St. Martin's Press, New York.

22. Nichols, T. E., Jr. 1983. Economic impact of aflatoxin in corn. South. Coop. Ser. Bull. 279:67-71.

23. Reddy, M. J., Shetty, H. S., Fanelli, C., and Lacey, J. 1992. Role of seed lipids in Aspergillus parasiticus growth and aflatoxin production. J. Sci. Food Agric. 59:177-181.

24. Squire, R. L. 1981. Ranking animal carcinogens: A proposed regulatory approach. Science 214:877.

25. Stössel, P. 1986. Aflatoxin contamination in soybeans: Role of proteinase inhibitors, zinc availability, and seed coat integrity. Appl. Environ. Microbiol. 52:68-72.

26. Tubajika, K. M., Guo, B. Z., Russin, J. S., Brown, R. L., Cleveland, T. E., and Widstrom, N. W. 1995. Factors associated with resistance to aflatoxin production in maize. (Abstr.) Phytopathology 85:512.

27. Widstrom, N. W., McMillan, W. W., and Wilson, D. M. 1987. Segregation for resistance to aflatoxin contamination among seeds on an ear of hybrid maize. Crop Sci. 27:961-963.

28. Widstrom, N. W., Wilson, D. M., and McMillan, W. W. 1984. Ear resistance of maize inbreds to field aflatoxin contamination. Crop Sci. 24:1155-1157.

29. Wolf, M. J., Buzan, C. L., MacMasters, M. M., and Rist, C. E. 1952. Structure of the mature corn kernel. II. Microscopic structure of the pericarp, seed coat and hilar layer of dent corn. Cereal Chem. 29:334-348. 